\title{
Behavior of microdroplets in diffuser/nozzle structures
}

\author{
Jing Liu $\cdot$ Yit Fatt Yap $\cdot$ Nam-Trung Nguyen \\ School of Mechanical and Aerospace Engineering, Nanyang Technological University, \\ 50 Nanyang Avenue, Singapore 639798, Singapore.e-mail: mntnguyen@ntu.edu.sg
}

\begin{abstract}
This paper investigates the behavior of micro-droplets flowing in microchannels with a series of diffuser/ nozzle structures. Depending on the imposed flow direction, the serial structures can act either as a series of diffusers or nozzles. Different serial diffuser/nozzle microchannels with opening angles ranging from $15^{\circ}$ to $45^{\circ}$ were considered. A $2 \mathrm{D}$ numerical model was employed to study the dynamics of the microdroplet during its passage through the diffuser/nozzle structures. The deformation of the microdroplet was captured using a level set method. On the experimental front, test devices were fabricated in polydimethylsiloxane using soft lithography. T-junctions for droplet formation, diffuser/nozzle structures and pressure ports were integrated in a single device. Mineral oil with $2 \%$ w/w surfactant span 80 and de-ionized water with fluorescent worked as the carrier phase and the dispersed phase, respectively. The deformation of the water droplet and the corresponding pressure drop across the diffuser/ nozzle structures were measured in both diffuser and nozzle configurations at a fixed flow rate ratio between oil and water of 30 . The results show a linear relationship between the pressure drop and the flow rate. Furthermore, the rectification effect was observed in all tested devices. The pressure drop in the diffuser configuration is higher than that of the nozzle configuration. Finally, the pressure measured results with droplet and without droplet were analyzed and compared.
\end{abstract}

\section{Keywords}

Microdroplet; Diffuser/nozzle; T-junction; Microchannel

\section{Introduction}

Droplet manipulation is important in droplet-based micro-fluidics, which has a number of applications in life sciences and analytical chemistry. Droplet generation, mixing, sorting and transportation are the key manipulation tasks. A number of actuation concepts such as electrowetting, theromocapillarity, and magnetism have been reported. Manipulation using channel geometry in a pressure driven flow can be a simple approach without the need of other external energy sources.

Teh et al. (2008) reviewed the different concepts for droplet generation, separation, mixing and their applications. Most of the reported concepts are based on the control of the surface tension or interfacial tension of droplets. For instance, Burns et al. (1996) employed a temperature gradient to move discrete nanoliter droplets through enclosed microchannels. Darhuber et al. (2003) used an addressable microheater arrays to handle liquid droplets on a planar surface. Nguyen and Huang (2005) investigated the effect of a transient temperature field 
on the droplet movement inside a cylindrical capillary. Besides thermocappillarity, microdroplet can also be manipulated by electrostatic and magnetic forces. While electrostatic force decreases the interfacial tension of the droplet at the contact line between the phases (Beni and Hackwood 1981), magnetic forces act as a body force on the whole droplet. Pollack and Fair (2000) used electrowetting as an actuation concept for manipulation of discrete microdroplets. Cho et al. (2003) demonstrated the different manipulation functions with electrowetting: generation, transporting, cutting, and merging. In terms of magnetic actuation, Safarik and Safarikova (1999) reviewed various strategies for separation of beads in a magnetic field. Lehmann et al. (2006) reported manipulation of droplets with dispersed magnetic microparticles. Basic functions such as merging, mixing, and splitting were demonstrated. Nguyen et al. (2006, 2007a) applied the same concept for manipulating ferrofluid droplets.

Serial diffuser/nozzle structures have been used as a flow rectification element for pumping applications in continuous-flow microfluidics. Stemme (1993) reported for the first time the use of this structure in valveless micro-pump. In a diffuser/nozzle structure with a small opening angle, the pressure loss in the diffuser configuration is lower than that of the nozzle configuration for an identical flow rate. However, a diffuser/nozzle structure with a large opening angle such as that reported by Gerlach and Wurmus (1995) showed the opposite behavior. The pressure drop in nozzle configuration is lower. A cut-off angle of around $10^{\circ}$ was proposed by Gerlach (1998). Gerlach also discussed the relationship between the rectification efficiency and the opening angles. Olsson et al. (2000) explained the behavior of small opening angle based on the pressure recovery in the expansion direction. At a large opening angle, the "vena-conracta" effect leads to flow separation and change the behavior of flow in both directions. All of the previous works are based on a single diffuser/nozzle element at high Reynolds number. The flow situation of inlet and outlet is different from which a serial of diffuser/nozzle elements are employed. The behavior of a series of diffuser/nozzle structures was not investigated in details before. The influence of opening angle on the rectification behavior of a diffuser/nozzle structure was investigated experimentally and numerically by Heschel et al. (1997) and Olsson et al. (2000), respectively. In all previous works, the width of the small opening and the length of the structure were fixed. However, the separation behavior of the flow also depends on the length of the structure. Nguyen and Huang (2001) used this concept to design and fabricate a micropump based on printed circuit board (PCB) technology. Recently, Groisman and Quake (2004) and Nguyen et al. (2008) reported that the rectification effect is even stronger if a viscoelastic fluid is employed instead of a Newtonian fluid. To the best knowledge of the authors, none of the previous works investigated multiphase flow in a serial diffuser/nozzle structure.

This paper investigates the behavior of a single droplet in a serial diffuser/nozzle microchannel. Three different opening angles $=15^{\circ}, 30^{\circ}$ and $45^{\circ}$ were considered. The design of diffuser/nozzle structures reported here is different than all other previous works. The widths of the small and large opening were fixed, while the length of the structure was varied to change the opening angle. The deformation of the droplet during its passage through the serial diffuser/nozzle microchannel is modeled numerically and measured using a microscope setup. The macroscopic behavior of the two phase flow was evaluated with the measurement of the pressure drop using the pressure ports integrated in the test devices. The results presented here showed potential applications in pumping of a two-phase system (e. g. a droplet train) and 
rheometry.

\section{Design and fabrication of test devices}

Figure 1a shows the layout of the device used in the investigations reported by this paper. The test section is a series of ten identical diffuser/nozzle structures. All structures have the same depth of $50 \mu \mathrm{m}$. Two pressure ports are located at the two ends of the test section to allow measurement access to a differential pressure sensor. Two T-junctions are used for the formation of droplets in two directions. Figure $1 \mathrm{~b}$ shows the dimensions of the T-junction used in our later experiments. Water as the dispersed phase is injected through a $40-\mu \mathrm{m}$ wide microchannel into the carrier channel with a width of $150 \mu \mathrm{m}$. Figure 2 depicts the detailed geometries of the different serial diffuser/nozzle microchannels used. For a fixed width of the diffuse/nozzle structures, channels with larger opening angle are shorter. The detailed geometries are shown in. Fig. 2. Each test device measures $10 \mathrm{~mm} \times 10 \mathrm{~mm}$.

The test devices were fabricated in polydimethylsiloxane (PDMS) using the soft lithography technique as described in Fig. 3. The layout depicted in Fig. 1a was designed using AutoCad and printed on a transparency film using a laser printer with a resolution of 8,000 dpi. The transparency mask was subsequently used for defining the negative mold of the devices in SU-8, Fig. 3b. For this purpose, a 50- $\mu \mathrm{m}$ thick SU-8 layer was first spin-coated on a silicon wafer, Fig. 3a. The SU-8 layer was then exposed and developed, Fig. 3c. Figure $3 \mathrm{~g}$ shows the SEM image of the SU-8 mold of one of the devices. PDMS was mixed from the two components with a weight ratio of 10:1, and then poured into the SU-8 mold, Fig. 3d. Air bubbles were removed using a vacuum oven at $60^{\circ} \mathrm{C}$. The PDMS part was left at room temperature for $24 \mathrm{~h}$ to cure. The PDMS was then peeled off from the master mold, Fig. 3e. The six ports with a diameter of $0.5 \mathrm{~mm}$ were punched using a special tool. The molded PDMS part with the microfluidic structures was subsequently bonded to another flat PDMS piece after treating both surfaces with oxygen plasma, Fig. $3 \mathrm{f}$.

\section{Numerical simulation}

\subsection{Problem description}

The numerical simulation was carried out with a two-dimensional (2D) model. Although a 3D model describes the problem more accurately, a 2D model for the relatively low aspect ratio of our case gives sufficient qualitative insights into the physics of the problem at a reasonable computing time. The dimensions of the microchannels follow those of the fabricated devices as depicted in Fig. 2. All three designs with different opening angles $\alpha=15^{\circ}, 30^{\circ}$ and $45^{\circ}$ were considered. In all devices, the straight microchannels outside of the test section have the same width of $150 \mu \mathrm{m}$. In order to reduce computational time, instead of ten only three diffuser/nozzle structures were considered in the simulation.

Two phases, the carrier fluid (mineral oil) and the dispersed fluid (water), are involved in the simulation. In the later experiments, the carrier fluid (oil) always wet the channel wall. An oil film exists between the droplet (water) and the channel wall. Thus the contact angle at the wall/oil/water contact line is neglected. The droplet is pressure driven via the immiscible carrier 
fluid and squeezed through the diffuser/nozzle structures. The hydrodynamic force of the carrier fluid flow exerts on the droplet in the form of surface stress. Driven by the surface force, the droplet moves and simultaneously deforms.

At the inlet, the carrier flow is assumed to be fully developed with an average velocity given by

$$
\bar{u}=\frac{Q}{W H}
$$

where $Q$ is the volumetric flow rate set by the syringe pump, $W$ is the channel width, and $H$ is the channel height. No-slip condition is enforced at the walls. Since the channel is oblique and the uniform grid is used, the boundary conditions are carried out with the block off approach of Patankar (1980). Outflow boundary condition is used at the exit.

In the present article, the Reynolds number is defined as

$$
\operatorname{Re}=\frac{2 \rho Q}{\mu(H+W)}
$$

where $\mu$ is dynamic viscosity and $\rho$ is density of the carrier fluid. In the conducted experiments, the maximum Reynolds number is approximately 0.032 corresponding to the highest oil flow rate of $300 \mu \mathrm{l} / \mathrm{h}$. The $R e$ is low enough to warrant an assumption of laminar flow. The circular/elliptical droplet is centered at $\left(x_{\mathrm{c}}, y_{\mathrm{c}}\right)$. The initial diameter of the droplets were extracted from the images captured using a (coupled charge device) CCD camera during the experiments. A program was written in MATLAB for this purpose. Simulations were performed for flow rate ratios between water and oil of 3:90 $\mu \mathrm{l} / \mathrm{h}, 6: 180 \mu \mathrm{l} / \mathrm{h}$, and 9:270 $\mu \mathrm{l} / \mathrm{h}$ for both configurations with different droplet sizes. It is worth noted that the flow rate ratio is fixed at 1:30. This large flow rate ratio is selected to ensure that there is only one droplet in the entire test section at any given time. With this, complex dynamical interactions between microdroplets are avoided, making the investigation more straightforward. The measurement results can then be compared to the simulation results.

\subsection{Governing equations and solution procedure}

There are a total of two-phases in the domain of interest, i.e. water and oil, separated by an interface $\Gamma$. To distinguish these phases, the level-set function (Osher and Sethian 1988) is employed. This function is defined as a signed distance function from the interface with the following sign convention,

$$
\phi(\bar{x}, t)= \begin{cases}>0, & \bar{x} \in \text { water } \\ 0, & \bar{x} \in \Gamma \\ <0, & \bar{x} \in \text { oil }\end{cases}
$$

Figure 4 shows the contours of the level set function. Driven by the underlying flow field, the 
interface $\Gamma$ evolves, changing the region occupied by oil and water. The level-set function is evolved based on the relation:

$$
\frac{\partial \phi}{\partial t}+u \frac{\partial \phi}{\partial x}+v \frac{\partial \phi}{\partial y}=0
$$

with $u$ and $v$ are the velocity components in the $x$ and $y$ directions respectively.

With this, the density and viscosity of the fluid in the domain at any instant $t$ can be calculated from (Chang 1996)

$$
\begin{aligned}
& \rho=H \rho_{\mathrm{w}}+(1-H) \rho_{\mathrm{o}} \\
& \mu=H \mu_{\mathrm{w}}+(1-H) \mu_{\mathrm{o}}
\end{aligned}
$$

where the subscripts $\mathrm{w}$ and o refer to water and oil, and the smoothed Heaviside function $H$ is defined by

$$
H(\phi)= \begin{cases}0 & \phi<-\varepsilon \\ (\phi+\varepsilon) /(2 \varepsilon)+\sin (\pi \phi / \varepsilon) /(2 \pi) & |\phi|<\varepsilon \\ 1 & \phi>\varepsilon\end{cases}
$$

The Heaviside function is smoothed over a finite thickness of $2 \varepsilon$. The parameter $\varepsilon$ is usually taken as a factor of the grid spacing. It is set to 1.5 of the control volume thickness in a uniform grid.

The continuity and momentum equations in a 2D Cartesian coordinate system for unsteady, viscous, incompressible, immiscible two-phase systems can be expressed as,

$$
\begin{aligned}
\frac{\partial \rho}{\partial t}+\frac{\partial(\rho u)}{\partial x}+\frac{\partial(\rho v)}{\partial y}=0 & \\
\frac{\partial(\rho u)}{\partial t}+\frac{\partial(\rho u u)}{\partial x}+\frac{\partial(\rho v u)}{\partial y}= & -\frac{\partial p}{\partial x}+\frac{\partial}{\partial x}\left(\mu \frac{\partial u}{\partial x}\right) \\
& +\frac{\partial}{\partial y}\left(\mu \frac{\partial u}{\partial y}\right)+\frac{\partial \mu}{\partial x} \frac{\partial u}{\partial x}+\frac{\partial \mu}{\partial y} \frac{\partial v}{\partial x} \\
& +F_{\sigma x} \\
\frac{\partial(\rho v)}{\partial t}+\frac{\partial(\rho u v)}{\partial x}+\frac{\partial(\rho v v)}{\partial y}= & -\frac{\partial p}{\partial y}+\frac{\partial}{\partial x}\left(\mu \frac{\partial v}{\partial x}\right) \\
& +\frac{\partial}{\partial y}\left(\mu \frac{\partial v}{\partial y}\right)+\frac{\partial \mu}{\partial x} \frac{\partial u}{\partial y}+\frac{\partial \mu}{\partial y} \frac{\partial v}{\partial y} \\
& +F_{\sigma y}
\end{aligned}
$$

where $p$ is the pressure, $F_{\sigma}$ is the interfacial force at the interface per unit volume. The 
subscripts $x$ and $y$ correspond to the axis directions. The interfacial force can be implemented within the framework of the continuum surface force model of Brackbill et al. (1992) as

$$
\begin{aligned}
& F_{\sigma x}=-\sigma \delta(\phi) n_{x} \kappa \\
& F_{\sigma y}=-\sigma \delta(\phi) n_{y} \kappa
\end{aligned}
$$

where $\sigma$ is the coefficient of surface tension and the Dirac delta function $\delta(\phi)$ define as

$$
\delta(\phi)= \begin{cases}(1+\cos (\pi \phi / \varepsilon)) /(2 \varepsilon) & |\phi|<\varepsilon \\ 0 & \text { otherwise }\end{cases}
$$

In Eq. 9a, 9b, the components of the unit normal of the interface $\left(n_{x}, n_{y}\right)$ and the curvature $\kappa(\phi)$ are given by

$$
\begin{aligned}
& n_{x}=\frac{\partial \phi}{\partial x} /|\nabla \phi| \\
& n_{y}=\frac{\partial \phi}{\partial y} /|\nabla \phi| \\
& \kappa(\phi)=-\frac{\phi_{y}^{2} \phi_{x x}-2 \phi_{x} \phi_{y} \phi_{x y}+\phi_{x}^{2} \phi_{y y}}{\left(\phi_{x}^{2}+\phi_{y}^{2}\right)^{3 / 2}}
\end{aligned}
$$

The governing equations are solved by a finite volume method (Patankar 1980) on a staggered mesh via the algorithm outlined in Yap et al. (2006). The wall is treated with the block-off approach of Patankar (1980). In this approach, the oblique wall is modeled as a staggered staircase-like profile. However, upon refining the mesh, the stair-case approximation of the wall approaches that of a smooth oblique wall. The mesh was refined sufficiently so that the solution is no longer affected by the stair-case approximation of the wall.

\subsection{Grid independent test}

Numerical simulations were performed on a uniform grid. Figure 5 shows grid independent study for a nozzle configuration with an opening angle of $\alpha=15^{\circ}$. Two different meshes of 470 $\times 40$ control volumes $(\mathrm{CVs})$ with a time step size of $\Delta t=4 \times 10^{-5} \mathrm{~s}$ and $940 \times 80 \mathrm{CVs}$ with $\Delta t=2 \times 10^{-5} \mathrm{~s}$ were used. The difference between the solutions of these meshes is small. Therefore, a mesh of $470 \times 40 \mathrm{CVs}$ with $\Delta t=4 \times 10^{-5} \mathrm{~s}$ is sufficient to capture all the essential flow features. The grid independent investigations were done similarly for other serial diffuser/ nozzle microchannels. Due to the space limitations, these will not be presented.

\subsection{Evaluation of pressure drop}

Figure 6 shows the time history of the simulated pressure drop across the diffuser/nozzle structures. The fluctuation is caused by the change in pressure drop when the droplet passages the sudden expansion or sudden constriction. According to our design the larger the opening 
angle the shorter is diffuser/nozzle structure. Consequently, the pressure drop at a larger opening angle is lower. A droplet at a throat blocks the flow and decreases the pressure drop. The lower pressure drop level shown in Fig. 6 corresponds to the situation when the droplet blocks the throat between two neighboring diffuser/nozzle structures. With an opening angle of $\alpha=15^{\circ}$, the droplets is small compared to the length of the structure and the three low-pressure periods can be clearly observed, Fig. 6a. In structures with larger opening angles of $\alpha=30^{\circ}$ and $\alpha=45^{\circ}$, the droplet is larger compared to the length of the structure and can occupy two throats at the same time. This situation leads to two low-pressure periods shown in Fig. 6b, c.

\section{Experiments}

\subsection{Materials}

In the experiment, de-ionized (DI) water and mineral oil work as the dispersed phase and the continuous phase, respectively. DI water was mixed with $0.01 \% \mathrm{w} / \mathrm{w}$ fluorescence dye (fluorescein disodium salt $\mathrm{C}_{20} \mathrm{H}_{10} \mathrm{Na}_{2} \mathrm{O}_{5}$, Acid Yellow 73 or CI 45350) to achieve better visualization with fluorescent microscopy. To reduce the interfacial tension between water and oil, $2 \%$ w/w surfactant span 80 was added to the oil. At a lower interfacial tension, its easier to form small water droplets even at relatively low oil flow rates. The dynamic viscosity of mineral oil and the interfacial tension between these two immiscible liquids were measured at $25^{\circ} \mathrm{C}$ as $23.8 \mathrm{mPas}$ and $3.65 \mathrm{mN} / \mathrm{m}$, respectively (Nguyen et al. 2007b).

\subsection{Experimental setup}

Figure 7a shows the schematic of the experimental setup. The working liquids were kept in 5$\mathrm{ml}$ glass syringes. Each syringe was driven by an individual syringe pump (KDS230, KD Scientific Inc., USA) to allow any flow rate ratio needed for the experiments. The pumps are connected in daisy-chain mode and can be controlled by a single serial port from the personal computer (PC). Hard Teflon tubes connected the syringes with the inlets of the microfluidic chip, Fig. 7b. The inlets of the microfluidic chip were made of stainless steel needles. The needle has an inner diameter of $0.33 \mathrm{~mm}$ and an outer diameter $0.64 \mathrm{~mm}$, that is press fit into the $0.5-\mathrm{mm}$ access hole previously punched into the PDMS substrate. The flow rate ratio was controlled by changing the volumetric flow rate of the pumps. The deformation of the droplet in the diffuser/nozzle microchannel was recorded with imaging software on the PC. As shown in Fig. 7b, the test device was placed on the inverted microscope (Nikon EclipseTE 2000-S, Japan). Both phase-contrast and fluorescent modes were used to capture the image of the droplets. A sensitive CCD camera (HiSense MKII) attached to the microscope was used to capture the droplet image. In addition to the CCD camera, droplet images were also recorded using a commercial digital camcorder (DCR-DVD803E, Sony, Japan) attached to the eyepiece of the microscope. The pressure ports on the chip were connected to a pressure sensor (HCX001, Sensortechnics, Germany). The pressure sensor has a linear range from 0 to 1 bar corresponding to an output voltage ranging from 0.5 to $4.5 \mathrm{~V}$. The sensor requires a calibrated supply voltage between 4.8 and $15 \mathrm{~V}$. A digital oscilloscope (TD220, Tektronix, USA) was used for collecting the electrical signal and converts it to a waveform, which was subsequently transferred to the PC via the serial interface. In our experiments, the dynamics of the pressure measurement system cannot follow the changes in pressure drop as shown in Fig. 6. Thus, the 
recorded data is interpreted as the average pressure across the test section.

Figure $1 \mathrm{~b}$ shows how a water droplet was formed at the T-junction. As mentioned above, the large flow rate ratio of 30 ensures that only one water droplet passing through the whole diffuser/nozzle microchannel at a given time. Since the flow rate ratio is fixed, the droplet size is adjusted by the flow rates of both oil and water. A settling time of at least 10 min was allocated for the flow to stabilize after changing the flow rates.

The pressure drop was measured in both configurations at different flow rates of oil. At a fixed flow rate ratio, the oil flow rate was varied from 30 to $300 \mu \mathrm{l} / \mathrm{h}$ corresponding to a water flow rate ranging from 1 to $10 \mu \mathrm{l} / \mathrm{h}$. Hence, ten set data are measured. The pressure data is considered as stable, if the standard deviation between two sets of data averaged over an interval of $10 \mathrm{~min}$ is $<1 \%$. The pressure drop without droplets was also measured as a reference. Five data sets are considered. The flow rate between water and oil are 2:60 $\mu \mathrm{l} / \mathrm{h}, 4: 120 \mu \mathrm{l} / \mathrm{h}$, and $6: 180 \mu \mathrm{l} / \mathrm{h}, 8: 240 \mu \mathrm{l} / \mathrm{h}, 10: 300 \mu \mathrm{l} / \mathrm{h}$.

\section{Results and discussion}

Tables 1, 2, 3 compare the droplet shapes obtained numerically and experimentally in structures with opening angles of $\alpha=15^{\circ}, 30^{\circ}$ and $45^{\circ}$. Oil flow rates of 90,180 , and $270 \mu 1 / \mathrm{h}$ were considered. During the experiment, the deformed droplets have highly repeatable. Due to the high speed of droplet flowing in the microchannel, the camera that we are using does not have sufficient temporal resolution to capture the deformation of a particular droplet in one droplet movement cycle. The deformed droplets shown in the tables are not the same droplet for each case. A direct temporal comparison between simulation results experimental results is not possible. The agreement between simulation and measurement shows that the numerical method can describe well the deformation of a droplet in a diffuser/nozzle structure. The experimental droplets size show the same trend for all opening angles that the droplet sizes is smaller at a higher flow rate ratio, Fig. 8. This behavior is consistent with previous observation of droplet formation at a T-junction (Garstecki et al. 2006). The larger shear rate caused by a higher flow rate causes the droplet to breakup earlier leading to a smaller droplet. Figure 9 depicts the droplet size as function of oil flow rate at the same flow rate ratio of 30 . The higher the flow rate, the smaller is the droplet size.

Comparing the droplet size formed in both directions shows that the droplet formed in diffuser direction is smaller than that formed in nozzle direction. This phenomenon can be explained by the higher fluidic resistance in diffuser direction (Garstecki et al. 2006), which is later confirmed by the measurement of the pressure drop. The average droplet diameter also decreases with increasing oil flow rate.

When the droplet moves through the constriction between two diffuser/nozzle elements, satellite droplets were formed at its tail. The sudden change of the static pressure at the constriction and the low interfacial tension used in the experiments are the reason for this breakup. The numerical model can not capture this phenomenon.

Since the Reynolds number is much smaller than 1 in the experiment, the nonlinear inertial 
term in the Navier- Stokes equation is not significant. Thus, the flow is Stokes flow and the pressure drop is linearly proportional to the flow rate. The measured results reflect this relationship which is depicted in Fig. 10 with the opening angles of $\alpha=15^{\circ}, 30^{\circ}$ and $45^{\circ}$. Both fluids with droplet and without droplet are presented. For the case without droplet, the magnitude of oil flow rate corresponds to the total flow rate in the case with droplet. The relative error increases with the increasing opening angles because of the shorter test structures and the corresponding lower pressure drop.

The concept of diodicity was used to quantify the rectification effect of the tested structures. For the Stokes flow at a low Reynolds number, the pressure drop and the flow rate have the relation

$$
\Delta p=C Q
$$

where $\mathrm{C}$ is the proportional factor representing the pressure loss across the structure. Here, we define the ratio between factors of diffuser direction and nozzle direction as the diodicity

$\zeta=C_{\text {diffuser }} / C_{\text {nozzle }}$

The diodicity Eq. 13 is independent of the flow rate. Figure 11 shows the diodicities for fluids with droplet and without droplet as a function of the opening angle $\alpha$. The values are slightly bigger than 1 and the maximum at $\alpha=15^{\circ}$ can be observed for both cases. Therefore, the rectification effect is not significant for our test structures. However, the existence of a droplet in the serial structures can slightly improve the rectification effect. If many droplets contained in the structure simultaneously, it may improve the diodicity further. If the structures are used in micropump, it indicates that the pumping direction is nozzle direction. This kind of micropump will be designed and tested in the future work.

The rectification effect in the Stokes regime at low Reynolds number observed here may have different reasons. Compared to previous works on stand-alone diffuser/nozzle structures, the inlet and outlet conditions of the diffuser/nozzle structures connected in series are different. Furthermore, our designs have fixed widths at the two ends of the diffuser/nozzle structure. The structure length changes with the opening angles. The longer structure may allow flow separation even at very low Reynolds number. A further possible reason for rectification behavior at low Reynolds number is the interaction with device material. The deformation of the microchannel channel due to the soft device material (PDMS) may also affect the pressure drop behavior.

\section{Conclusions}

In conclusion, we have reported numerical and experimental results on the behavior of microdroplets in an array of diffuser/nozzle structures. The deformation of a water droplet in diffuser/nozzle structures with opening angles ranging from $15^{\circ}$ to $45^{\circ}$ were investigated. A $2 \mathrm{D}$ numerical model was formulated and solved to track the deformation of a microdroplet during the passage through the diffuser/ nozzle structures. Level set method was used in this model to 
track the moving interface boundary. The test devices were fabricated in PDMS using soft lithography. The simulation and experimental results show that the numerical model can capture well the deformation of the droplet inside the diffuser/nozzle structure. Since the deformation is determined by the two key factors, the interfacial tension and the viscosity of the carrier fluid, the device and the experiment can potentially used for rheometry. At a constant flow rate ratio between oil and water, the experimental results show a linear relation between the pressure drop and the flow rates. A rectification effect was observed in all tested devices. Despite the low Reynolds number of less than 0.032, the pressure drop in diffuser direction is higher than in the nozzle direction. This effect is consistent with the behavior of a continuous flow of a Newtonian fluid through a diffuser/nozzle structure and can be used for designing micropumps for two-phase flow. In the future, we will focus on the improvement of the numerical model to properly describe the rectification effects as observed in the experiments. A pump with an oscillating pressure port between two diffuser/nozzle arrays will be able to deliver a droplet train. In many applications such as protein crystallization in microdroplets, an existing droplet train, immobile for the period of crystallization, will need a pump for on-chip transport and further processing. The current 2D model gives a qualitative insight into the physics of the problem. A 3D simulation gives us quantitative results for better comparison. Currently, we are working on the 3D numerical simulation of the problem. The 3D simulation results on relation between pressure changes and droplets deformation will be presented in the near future.

\section{Acknowledgments}

The authors gratefully acknowledge the support from the Agency of Science, Technology and Research (A*STAR), Singapore (grant number SERC 0521010108 "Droplet-based micro/ nanofluidics"). 


\section{References}

Beni G, Hackwood S (1981) Electro-wetting displays. Appl Phys Lett 38(4):207-209

Burns MA, Mastrangelo CH, Sammarco TS, Man FP, Webster JR, Johnson BN, Foerster B, Jones D, Fields Y, Kaiser AR (1996) Microfabricated structures for integrated DNA analysis. Science 93:5556-5561

Cho SK, Moon H, Kim CJ, Member IEEE, Member ASME (2003) Creating, transporting, cutting, and merging liquid droplets by electrowetting-based actuation for digital microfluidic circuits. J Microelectromech Syst 12(1):70-80

Darhuber AA, Valentino JP, Davis JM, Troian SM (2003) Microfluidic actuation by modulation of surface stresses. Appl Phys Lett 82(4):657-659

Garstecki P, Fuerstman MJ, Stone HA, Whitesides GM (2006) Formation of droplets and bubbles in a microfluidic T-junction - scaling and mechanism of break-up. Lab Chip 6:437446

Gerlach T (1998) Microdiffusers as dynamic passive valves for micropump applications. Sens Actuators A 69:181-191

Gerlach T, Wurmus H (1995) Working principle and performance of the dynamic micropump. IEEE pp 221-225

Groisman A, Quake SR (2004) A microfluidic rectifier: anisotropic flow resistance at low Reynolds numbers. Phys Rev Lett 92: 094501-1-094501-4

Heschel M, Mullerborn M, Bouwstra S (1997) Fabrication and characterization of truly 3-D diffuser/nozzle microstructures in silicon. J Microelectromech Syst 6(1):41-47

Lehmann U, Hadjidj S, Parashar VK, Vandevyver C, Rida A, Gijs MAM (2006) Twodimensional magnetic manipulation of microdroplets on a chip as a platform for bioanalytical applications. Sens Actuators B 117:457-463

Nguyen NT, Huang XY (2001) Miniature valveless pumps based on printed circuit board technique. Sens Actuators A 88:104-111

Nguyen NT, Huang XY (2005) Thermocapillary effect of a liquid plug in transient temperature field. Jpn J Appl Phys 94:1139- 1142

Nguyen NT, Ng KM, Huang XY (2006) Manipulation of ferro fluid droplets using planar coils. Appl Phys Lett 89:052509

Nguyen NT, Beyzavi A, Ng KM, Huang XY (2007a) Kinematics and deformation of ferrofluid droplets under magnetic actuation. Microfluid Nanofluid 3:571-579

Nguyen NT, Ting TH, Yap YF, Wong TN, Chai CK, Ong WL, Zhou JL, Tan SH, Yobas L (2007b) Thermally mediated droplet formation in microchannels. Appl Phys Lett 91:084102$1-084102-3$

Nguyen NT, Lam YC, Ho SS, Low CLN (2008) Improvement of rectification effects in diffuser/nozzle structures with viscoelastic fluids. Biomicrofluidics 2:034101

Olsson A, Stemme G, Stemme E (2000) Numerical and experimental studies of flat-walled diffuser elements for valve-less micro-pumps. Sens Actuators 84:165-175

Osher S, Sethian JA (1988) Fronts propagating with curvature dependent speed: algorithms based on Hamilton-Jacobi formulations. J Comput Phys 79:12-49

Pollack MG, Fair RB (2000) Electrowetting-based actuation of liquid droplets for microfluidic applications. Appl Phys Lett 77(11):1725-1726

Safarik I, Safarikova M (1999) Use of magnetic techniques for the isolation of cells. J Chromatogr B 722:33-53 
Stemme E (1993) A valveless diffuser/nozzle based fluid pump. Sens Actuators A 39:159-167

Teh SY, Lin R, Hung LH, Lee AP (2008) Droplet microfluidics. Lap Chip 8:198-220

Yap YF, Chai JC, Wong TN, Toh KC, Zhang HY (2006) A global mass correction scheme for the level-set method. Num Heat Transfer B 50:455-472 


\section{List of Figure}

Figure 1 Device design for investigation of microdroplets in diffuser/ nozzle structures: a layout; $b$ mechanism for formation of microdroplets

Figure 2 Investigated diffuser/nozzle structures: a $\alpha=15^{\circ} ; \mathbf{b} \alpha=30^{\circ}$; $\alpha=45^{\circ}$

Figure 3 Fabrication of microchannels with soft lithography: a spin coating a silicon wafer with SU-8; b UV exposure with a clear mask; c development of SU-8 mold; $\mathbf{d}$ pouring PDMS on the mold; e peeling off the PDMS; $\mathbf{f}$ surface treatment of two pieces of PDMS with oxygen plasma; $\mathbf{g}$ the fabricated mold

Figure 4 Contours of constant level set function at $t=0 \mathrm{~s}$

Figure $5 \quad$ Grid independent study with $\alpha=15^{\circ}$ in nozzle direction (solid line: $470 \times 40$ CVs with $\Delta t=4 \times 10^{-5} \mathrm{~s}$, dotted line: $940 \times 80 \mathrm{CVs}$ with $\Delta t=2 \times 10^{-5} \mathrm{~s}$ )

Figure 6 Pressure drop with time of three diffuser/nozzle elements. The oil flow rate is $270 \mu \mathrm{l} / \mathrm{h}$, the water flow rate is $9 \mu \mathrm{l} / \mathrm{h}: \mathbf{a} \alpha=15^{\circ}, \mathbf{b} \alpha=30^{\circ}, \mathbf{c} \alpha=45^{\circ}$

Figure $7 \quad$ Experimental setup: a the schematic; $\mathbf{b}$ the device with eight test chips

Figure $8 \quad$ Effect of flow rate ratio on droplet size (oil flow rate of $300 \mu \mathrm{l} / \mathrm{h}$ )

Figure 9 Effect of flow rate on droplet size (flow rate ratio of 30)

Figure 10 Comparison of pressure drop between fluid flow with droplet and without droplet: a $\alpha=15^{\circ} ; \mathbf{b} \alpha=30^{\circ}$; c $\alpha=45^{\circ}$

Figure 11 Diodicities as a function of the opening anglr $\alpha$ 


\section{List of Tables}

Table 1 Comparison of droplet shape between numerical data and experimental data for $\alpha=15^{\circ}$

Table 2 Comparison of droplet shape between numerical data and experimental data for $\alpha=30^{\circ}$

Table 3 Comparison of droplet shape between numerical data and experimental data for $\alpha=45^{\circ}$ 


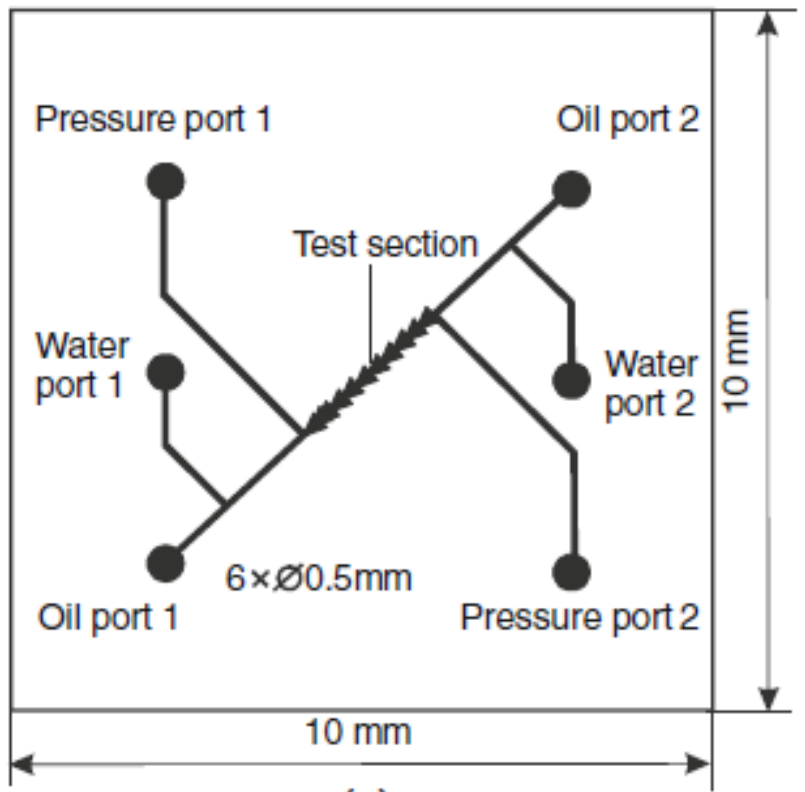

(a)

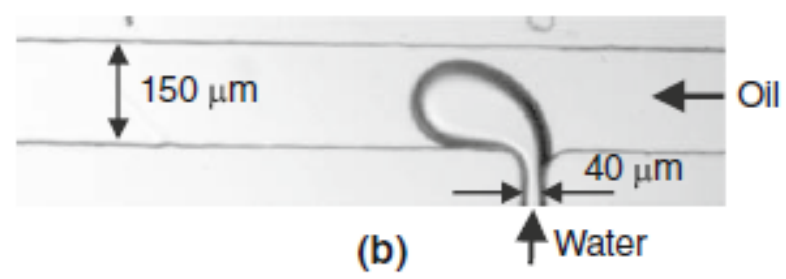

Figure 1 

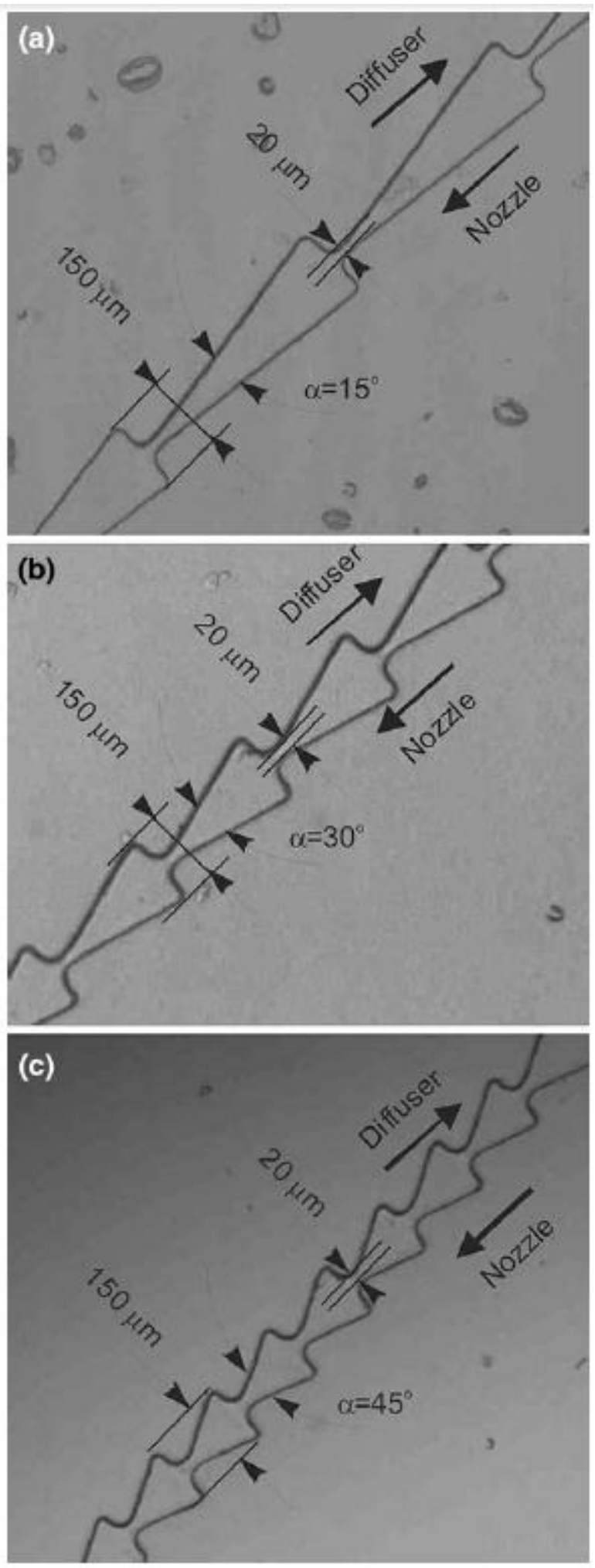

Figure 2 
(a) IIIIIIIIIIIIII

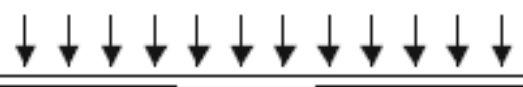

(b) mIIIIIIIIIIIIIII

(c) (1/1/1/11/1/1/1/

(d)

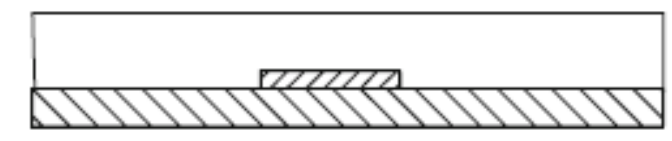

(e)

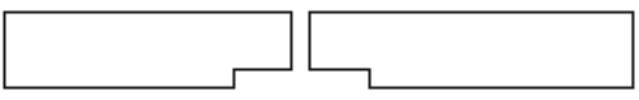

(f)

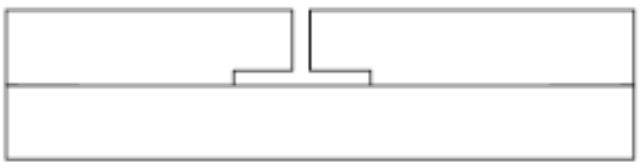

(g)

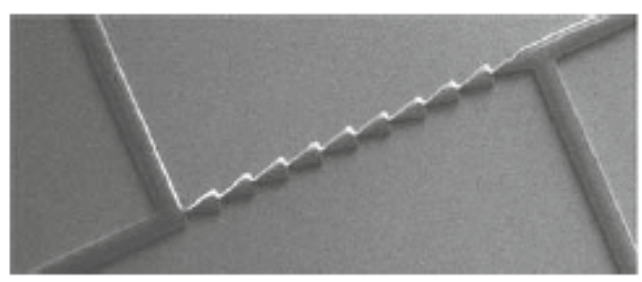

$\$ D$ Si PDID Su-8 $\square$ PDMS

Figure 3 


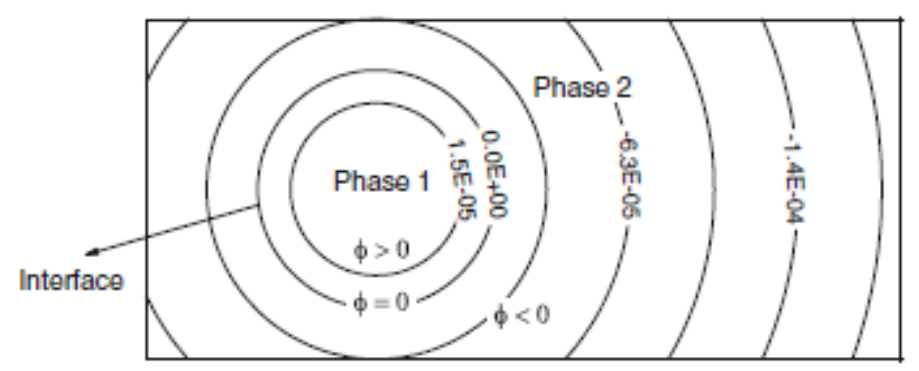

Figure 4 


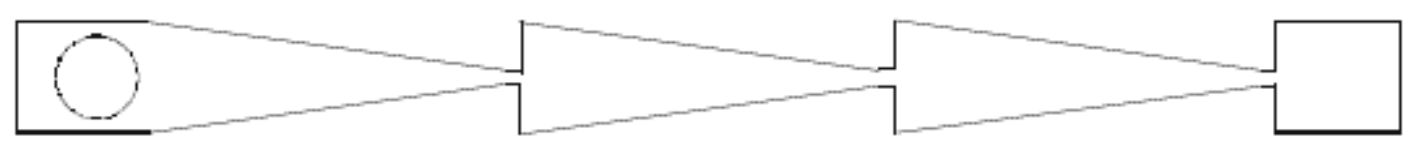

(a) t=0 ms

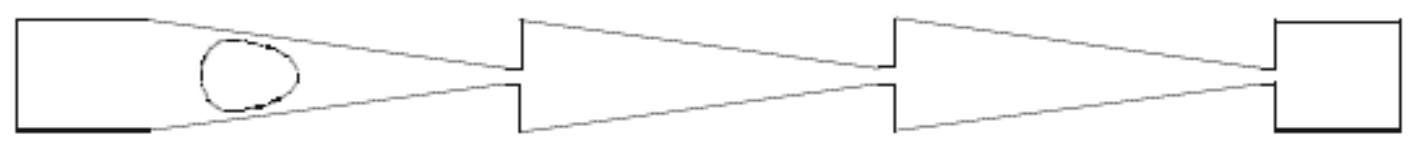

(b) $t=14.4 \mathrm{~ms}$

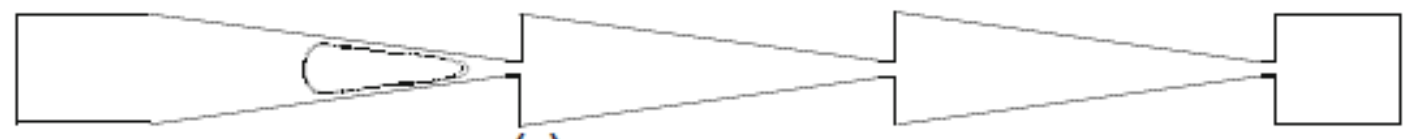

(c) $t=22.4 \mathrm{~ms}$

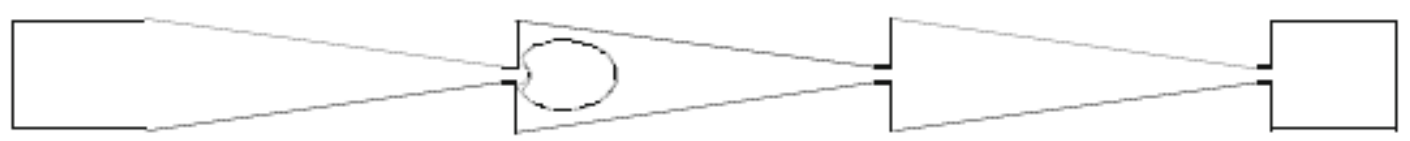

(d) $\mathrm{t}=30.4 \mathrm{~ms}$

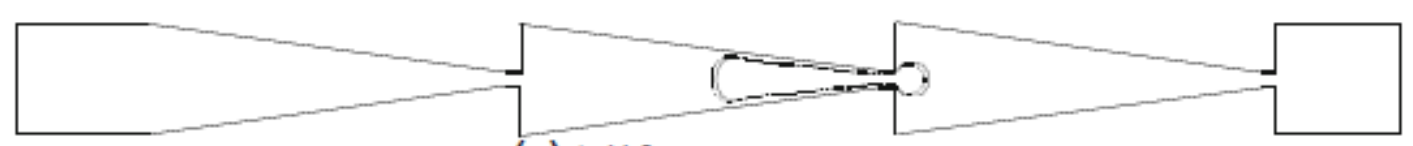

(e) $\mathrm{t}=44.8 \mathrm{~ms}$

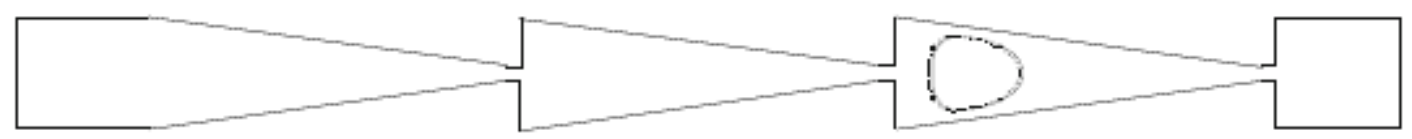

(f) $\mathrm{t}=52.8 \mathrm{~ms}$

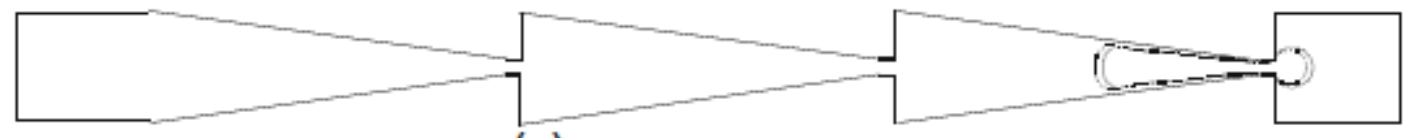

(g) $\mathrm{t}=65.6 \mathrm{~ms}$

Figure 5 

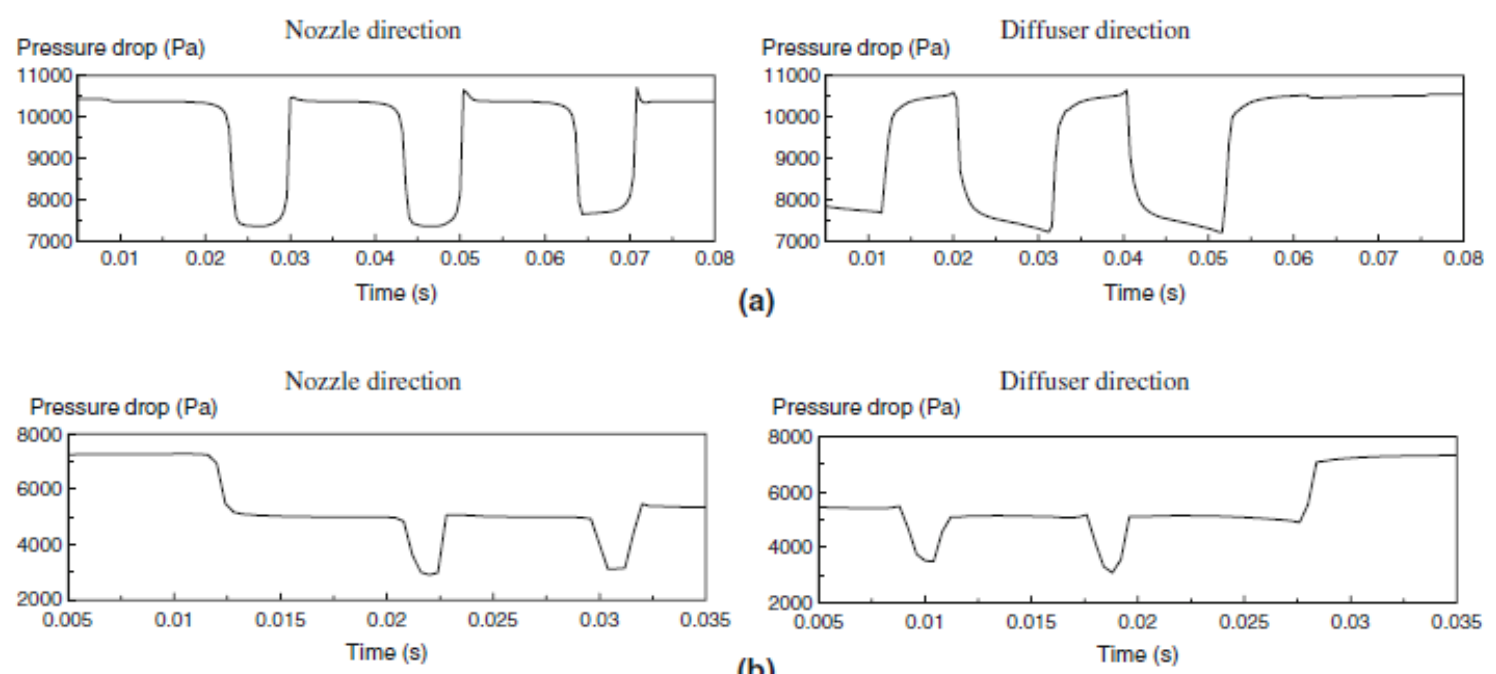

(b)
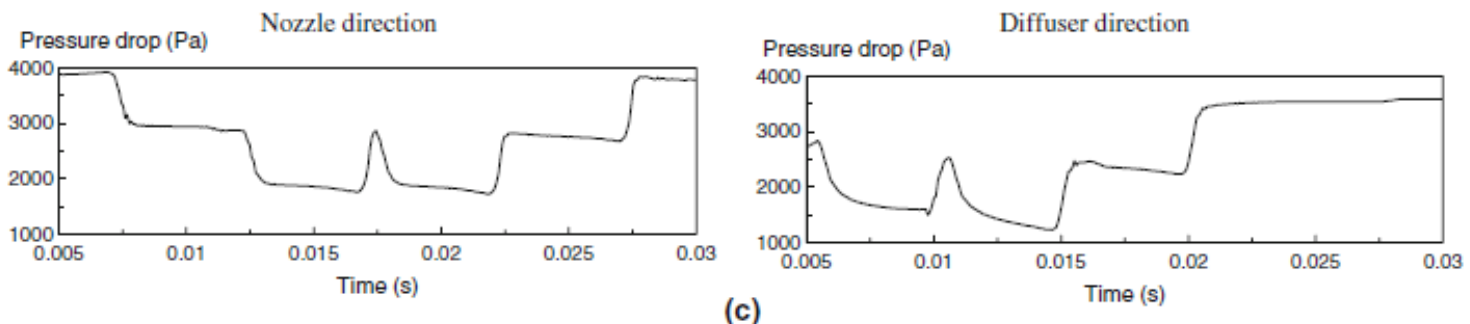

(c)

Figure 6 


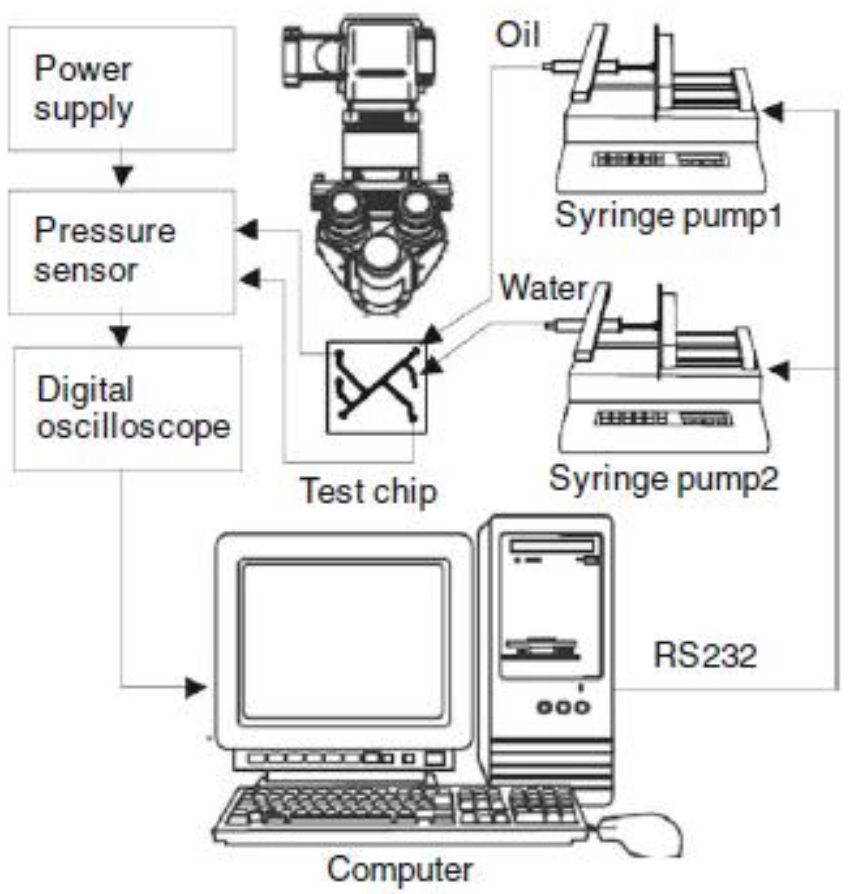

(a)

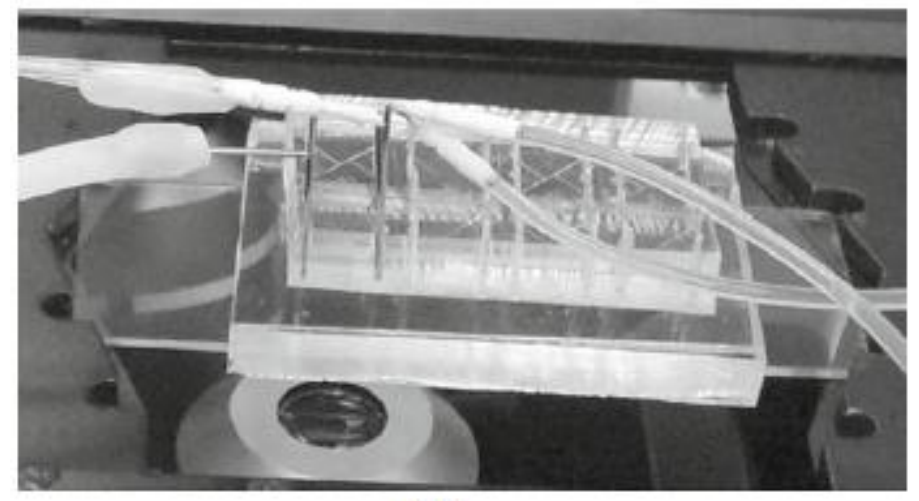

(b)

Figure 7 

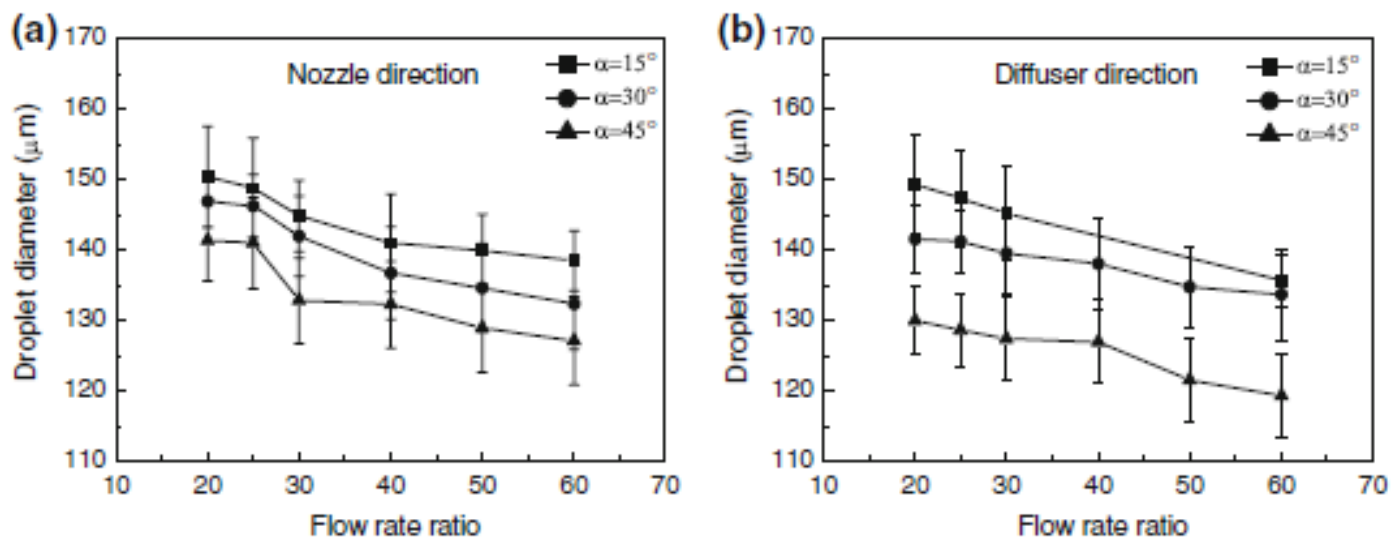

Figure 8 

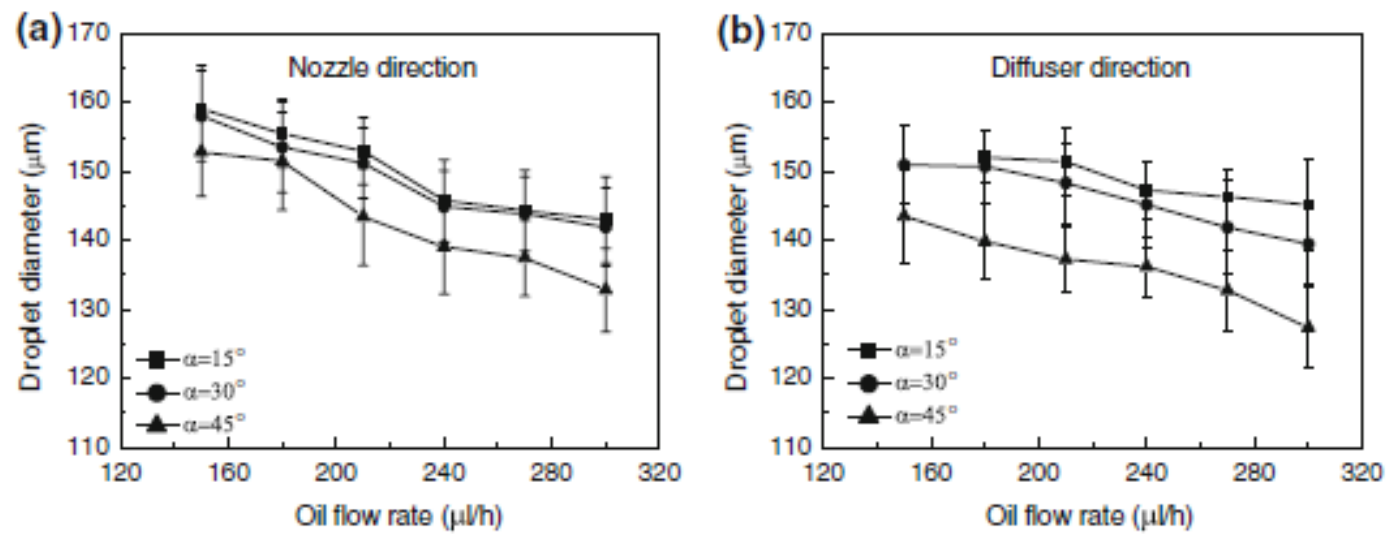

Figure 9 

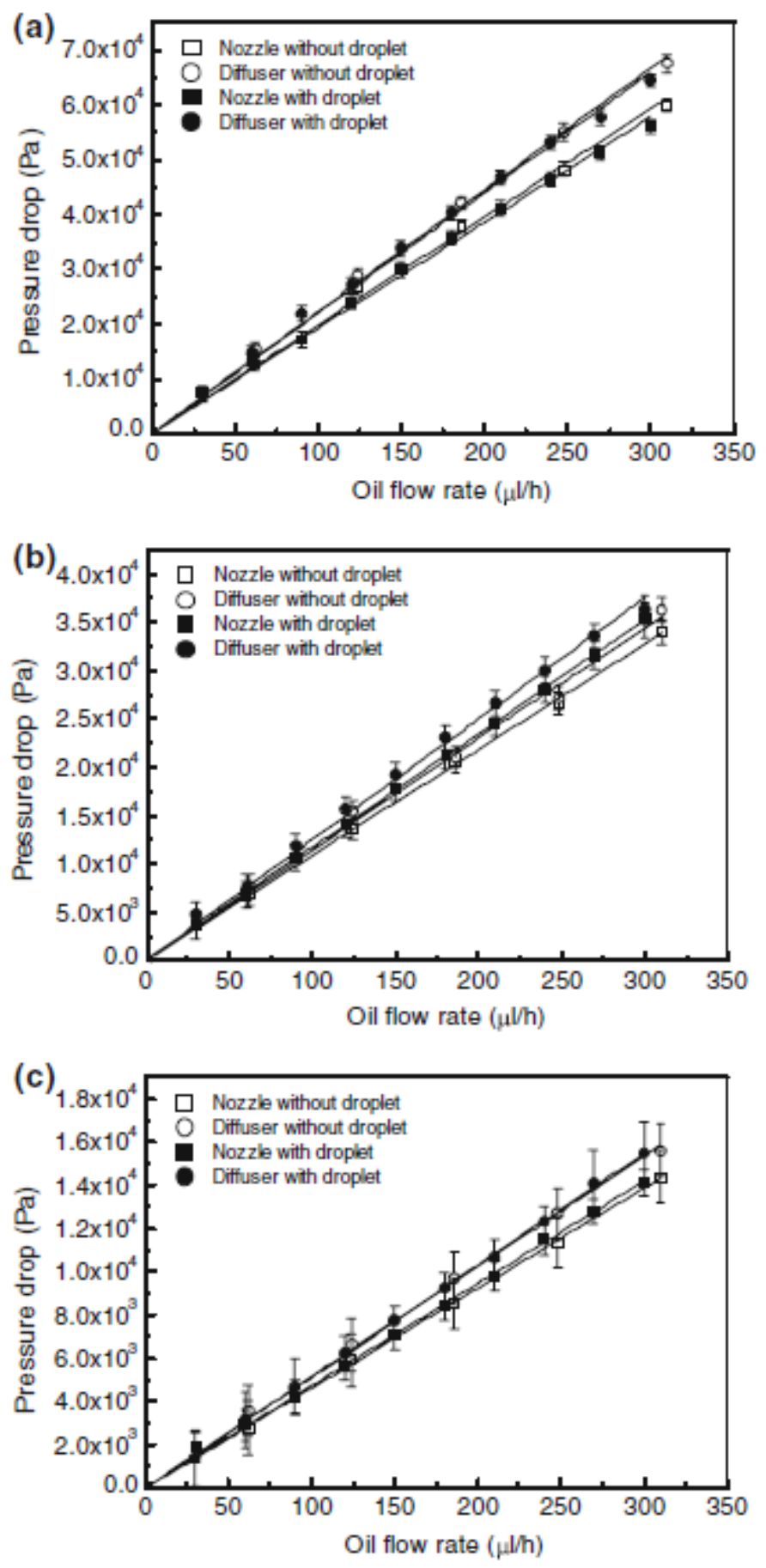

Figure 10 


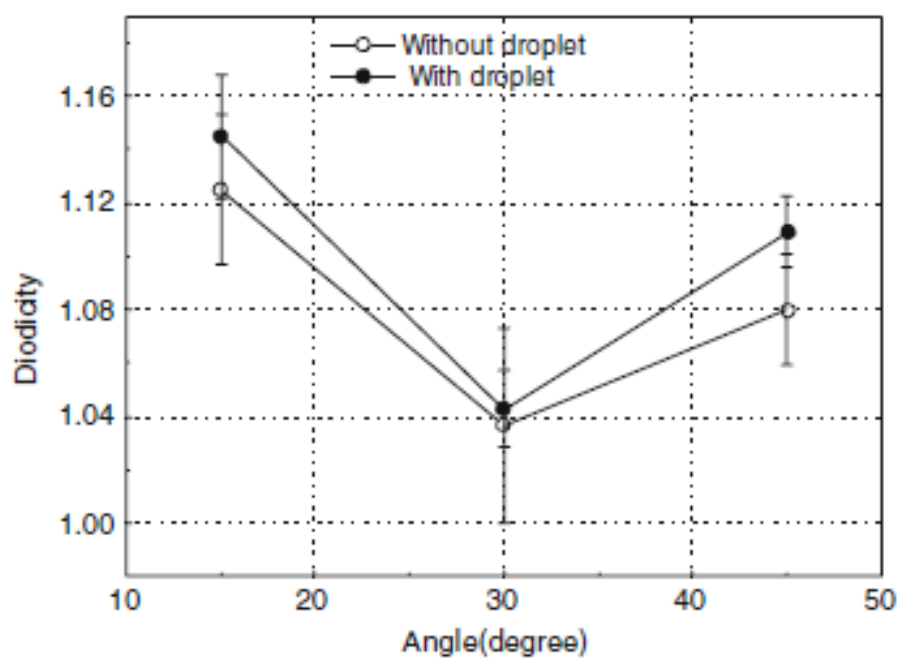

Figure 11 


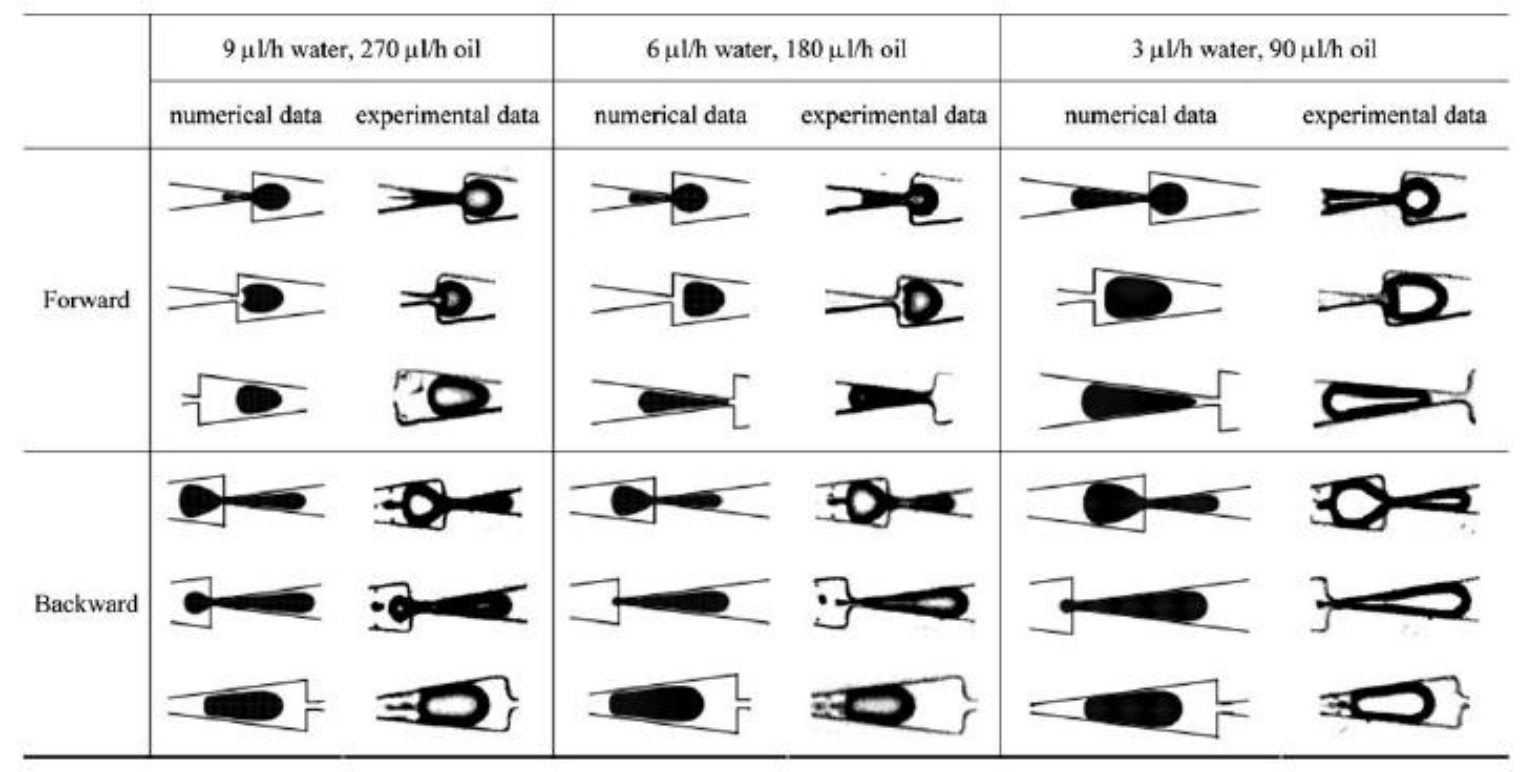

Table 1 


\begin{tabular}{|c|c|c|c|c|c|c|}
\hline & \multicolumn{2}{|c|}{$9 \mu \mathrm{l} / \mathrm{h}$ water, $270 \mu \mathrm{l} / \mathrm{h}$ oil } & \multicolumn{2}{|c|}{$6 \mu \mathrm{l} / \mathrm{h}$ water, $180 \mu \mathrm{l} / \mathrm{h}$ oil } & \multicolumn{2}{|c|}{$3 \mu / \mathrm{h}$ water, $90 \mu \mathrm{l} / \mathrm{h}$ oil } \\
\hline & numerical data & experimental data & numerical data & experimental data & numerical data & experimental data \\
\hline Forward & & & 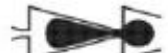 & 2 & & \\
\hline Backward & & & & & & \\
\hline & & & & & & \\
\hline
\end{tabular}

Table 2 


Forward

Table 3 